\title{
Investigation of photosensitivity of copolymer with (p-vinyl phenyl) cyclopropyl methyl cinnamate
}

\author{
Kazim Guliyev, Afat Aliyeva, Gulnara Ponomaryova, Abasgulu Guliyev, Dilbar Nurullayeva \\ Institute of Polymer Materials of Azerbaijan National Academy of Sciences, Sumgait, Azerbaijan
}

Email address:

ipoma@science.az (K. Guliyev),ndilbar@list.ru (D. Nurullayeva)

\section{To cite this article:}

Kazim Guliyev, Afat Aliyeva, Gulnara Ponomaryova, Abasgulu Guliyev, Dilbar Nurullayeva. Investigation of Photosensitivity of Copolymer with (P-Vinyl Phenyl) Cyclopropyl Methyl Cinnamate. American Journal of Applied Chemistry. Vol. 3, No. 1, 2015, pp. 21-24.

doi: 10.11648/j.ajac.20150301.14

\begin{abstract}
The radical copolymerization of (p-vinyl phenyl) cyclopropyl methyl cinnamate with methyl methacrylate has been studied. New cyclopropane-containing photosensitive copolymers have been prepared. The constant values of relative activity of monomers have been determined and the parameters of Q-e on Alfrey and Price have been calculated. The copolymerization constants of this compound (r1) with methyl methacrylate ( $\mathrm{r} 2$ ) calculated on Fineman-Ross method are $\mathrm{r} 1=1.05, \mathrm{r} 2=0.4$, respectively; parameter values $\mathrm{Q}$ and e: $\mathrm{Q} 1=2.5, \mathrm{e} 1=-0.9$, respectively. The composition and structure of these copolymers have been established. The photochemical investigations of the synthesized copolymer have been carried out. It has been established that a structuring proceeds due to opening of cyclopropane ring and carbonyl groups and also double bond in cinnamate fragment.
\end{abstract}

Keywords: Copolymerization, P-Vinylphenyl) Cyclopropyl Methylcinnamate, Photosensitivity

\section{Introduction}

For creation of negative photoresists it has been suggested a large number of compounds, however now only some of their representatives have found application. They include compositions based on photosensitive polymers containing groups, for ex., polyvinyl cinnamate and its derivatives. A review of these works is presented in [1]. For all types of photo-, electrono-resists there are a number of basic requirements, namely a high photosensitivity, satisfactory resolution, stability to chemical actions, good adhesion of the photoresist film for the substrate and good deformation-strength characteristics.

Due to this it is appeared a great interest of the researchers to preparation of new types of photosensitive polymers for microelectronics [2-8]. We have carried out a solution of this problem by polymerization of functionally substituted cyclopropyl styrenes [9-12].

The interest to preparation of such polymers has been stipulated, first of all, by the fact that in the forming macromolecules there are reactive functional groups of various nature as the suspensions in the basic macro chain. As a result of copolymerization of functional cyclopropane containing vinyl compounds being one of the perspective reactive monomers, there have been synthesized the polymers containing cyclopropane grouping regularly located in the side suspensions or macro chain $[13,14]$.

Table 1. Parameters of copolymerization of PCPC $\left(M_{1}\right)$ with $M M A\left(M_{2}\right)$.

\begin{tabular}{|c|c|c|c|c|c|c|c|c|c|c|c|c|}
\hline \multicolumn{2}{|c|}{$\begin{array}{l}\text { Composition of initial } \\
\text { mixture, mol.\% }\end{array}$} & \multirow{2}{*}{$\begin{array}{l}\text { B. } \\
\text { N., } \\
\text { G }\end{array}$} & \multicolumn{2}{|c|}{$\begin{array}{l}\text { Composition of } \\
\text { copolymers, mol.\% }\end{array}$} & \multirow{2}{*}{$r_{1}$} & \multirow{2}{*}{$\mathbf{r}_{2}$} & \multirow{2}{*}{$\mathbf{Q}_{1}$} & \multirow{2}{*}{$e_{1}$} & \multirow{2}{*}{$\mathbf{r}_{1} \cdot \mathbf{r}_{2}$} & \multicolumn{3}{|c|}{$\begin{array}{l}\text { Microstructure of } \\
\text { copolymer }\end{array}$} \\
\hline РСРС $\mathrm{M}_{1}$ & 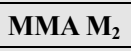 & & PCPC $\mathrm{m}_{1}$ & MMA $m_{2}$ & & & & & & $\mathbf{L}_{\mathbf{M}_{1}}$ & $\mathbf{L}_{\mathbf{M}_{2}}$ & $\mathbf{R}$ \\
\hline 10 & 90 & 7.7 & 19.36 & 80.64 & & & & & & 1.12 & 4.60 & 35 \\
\hline 25 & 75 & 15.0 & 37.90 & 62.10 & & & & & & 1.35 & 2.20 & 56 \\
\hline 50 & 50 & 23.5 & 59.35 & 40.65 & $1.05 \pm 0.03$ & $0.4 \pm 0.02$ & $2.5 \pm 0.01$ & $-0.9 \pm 0.02$ & 0.42 & 2.05 & 1.40 & 58 \\
\hline 75 & 25 & 31.1 & 78.54 & 21.46 & & & & & & 4.15 & 1.13 & 38 \\
\hline 90 & 10 & 36.0 & 90.91 & 9.09 & & & & & & 10.45 & 1.04 & 17 \\
\hline
\end{tabular}

$\mathrm{L}_{\mathrm{M}_{1}}$ and $\mathrm{L}_{\mathrm{M}_{2}}$ - average length of blocks of monomer links; $\mathrm{R}$ - Harwood blocking coefficient 
This work has been devoted to the investigation of copolymerization of (p-vinylphenyl)cyclopropyl methyl cinnamate (PCPC) with methyl methacrylate (MMA) and to the study of properties of copolymers prepared on their basis with the aim of creation of new photosensitive copolymers. The choice of this monomer has been stipulated by the fact that the concentration of double bonds and their chemical nature and also an availability of cyclopropane ring in combination with carbonyl group in monomer in resolution degree influence on such important photolithographic parameters of resists, as photosensitivity, adhesion, etc, as evidenced by a quite large experimental material accumulated up to present.

PCPC is the new reactive monomer, a formula and data about synthesis and homopolymerization of which are presnetd in work [12]. The choice of this compound for investigation of copolymerization with traditional monomer (MMA) has been stipulated by the availability of strongly absorbing light energy groups (carbonyl, double bond, cyclopropane) in a molecule of the synthesized monomer. For copolymerization of the studied systems, it is important to choose the conditions under which the polymerization would proceeded only on the vinyl group, and the reactive fragments would be remained in the side chain unchanged.

\section{Experimental}

The synthesis of PCPC was carried out according to the methodology described in work [12].

The copolymerization of the synthesized PCPC with MMA was carried out in the ampoules in benzene solution in the presence of $0.5 \%$ dinitrilazoisobutyric acid (AIBN) (from total mass of monomer) at $70^{\circ} \mathrm{C}$. Total concentration of the initial monomers was constant and was $1 \mathrm{~mol} / \mathrm{l}$ and a ratio of the initial monomers was changed in the concentrations shown in Table 1. The forming copolymer was purified by twofold reprecipitation from benzene solution to methanol and dried in vacuum (15-20 mm merc.c) at $30^{\circ} \mathrm{C}$ to constant mass. The copolymer being a powder of white color is well soluble in aromatic and chlorinated hydrocarbons. The characteristic viscosity was determined in Ubbelohde viscometer. The characteristic viscosity $([\eta])$ was $0.95-1 \mathrm{dl} / \mathrm{g}$.

The composition of copolymer was determined by analysis of functional groups (determination of double bonds on bromine number).

The IR-spectra of copolymers were registered on spectrometer UR-20, PMR spectra - on spectrometer BS-487B Tesla $(80 \mathrm{MHz})$ in a solution of deuterated chloroform.

For determination of photosensitivity of the synthesized polymer some compositions of copolymer at various concentrations $(4-13 \%$ solution) has been made. The application of layer of photoresist on substrates was carried out in the dust-free medium.

All solutions of resists applied on glass substrates by means of centrifuge at $2500 \mathrm{rev} \cdot \mathrm{min}^{1}$. After application the samples with photoresist are sustained for not less than $20 \mathrm{~min}$. for increase of photoresist adhesion to substrate. Then it is cut the photoresist on contour of the sample not permitted delamination of the film.

The thickness of the prepared film-resists was measured by microinterferometer "LINNIKA". The resist layer thickness after its drying for $10 \mathrm{~min}$. at room temperature and for $20 \mathrm{~min}$. at $40-45^{\circ} \mathrm{C} / 10 \mathrm{~mm}$ merc.c was $0.20-0.25 \mathrm{mcm}$.

The exposition of the samples with applied photoresist was carried out on the installation with point light source through photomask. As a source of UV-irradiation it was used a mercuric lamp DPT-220 (current force - 2.2 A, distance from radiation source $-15 \mathrm{~cm}$, mobile shutter rate of exposure meter $-720 \mathrm{~mm} \cdot \mathrm{h}^{-1}$, exposition time $-5-20 \mathrm{sec}$.). The development was carried out in the jet installation. As a developer it was used a mixture dioxane:isopropyl ether (1:2) at temperature $18-25^{\circ} \mathrm{C}$.

A criterion of photosensitivity of negative photoresists at UV-irradiation is the completeness of passing of the photochemical polymerization (cross-linking) reaction of molecules of photoresist base.

After exposition and development the content of insoluble polymer was calculated on residue mass as a fact of formation of cross-linked product.

Light sensitivity - value, inverse for dose of UV-light absorbed by the photoresist, in other words, the dose necessary for transfer of photoresist to insoluble state. It is measured in $\mathrm{cm}^{2} / \mathrm{Wt} \cdot \mathrm{s}=\mathrm{cm}^{2} / \mathrm{J}$ :

$$
\mathrm{S}=\frac{1}{\mathrm{H}}=\frac{1}{\mathrm{E} \cdot \mathrm{t}}
$$

$\mathrm{H}$ - exposition (or dose irradiated by UV-light), $\mathrm{J} \cdot \mathrm{cm}$

$\mathrm{E}$ - intensity, $\mathrm{Wt} / \mathrm{cm}^{2}$

$\mathrm{t}$ - duration of irradiation, $\mathrm{s}$

\section{Results and Discussion}

With the aim of establishment of structure of the synthesized copolymer the spectral analyses (IR- and PMR-spectroscopy) of the synthesized monomer and polymer samples have been carried out.

In view of the fact that PCPC is the polyfunctional compound, at its radical copolymerization with MMA it should be expected the formation of new reactive polyfunctional copolymer.

It has been established by comparison of IR-spectra of copolymers with spectra of the initial monomer (PCPC) that the absorption bands at 990 and $1640-1645 \mathrm{~cm}^{-1}$, referring to the deformation and valence vibrations of double bond of vinyl group disappear after copolymerization. The absorption bands characteristic for benzene ring and cyclopropane group are kept after polymerization. The absorption bands at $1720-1725,1030-1035$ and $1110 \mathrm{~cm}^{-1}$, referring vibrations cyclopropane, carbonyl and ether bond remain, correspondingly, unaffected. In the spectrum of copolymer 
there is also a band $1635 \mathrm{~cm}^{-1}$, which characterizes double bond of cinnamic acid.

In additon, in the range of frequencies of $800-680 \mathrm{~cm}^{-1}$ and at $780,750,730$ and $680 \mathrm{~cm}^{-1}$ the absorption bands characterizing monosubstituted benzenes in ethers of cinnamic acid were observed. The IR-spectrum of copolymer of PCPC with methyl methacrylate is presented in Fig. 1a (non-irradiated film).

In the PMR-spectrum of copolymer the resonance signals, referring to protons of benzene ring $(\delta=6.60-7.30 \mathrm{ppm})$ and cyclopropane ring $(\delta=0.65-1.66 \mathrm{ppm})$ are clearly appeared, and the signals, referring to protons of vinyl group $(\delta=$ 5.10-6.65 ppm) - are absent. According to the data of spectroscopy the copolymerization of PCPC with MMA proceeds only due to opening of double bonds of vinyl groups with keeping of other reactive functional fragments (phenyl cyclopropane and cinnamate). Thus, on the basis of analysis of the IR- and PMR-spectra of copolymers prepared by copolymerization of PCPC with MMA the following structure of copolymer is assumed:

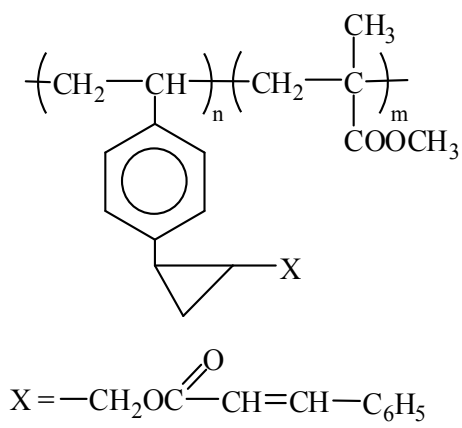

The copolymerization was carried out at various ratios of the initial monomers.

An initial composition is the main factor determining the characteristics of the polymers. It has been revealed that the composition of the forming copolymers depends on composition of the initial monomer mixture, ratio of activities of monomers and their radicals.

For estimation of polymerization activity of PCPC the constant values of relative activity of monomers on compositions of the initial monomer mixture on Fineman-Ross method [15] and parameters Q-e - on Alfrey and Price have been calculated. The parameters of microstructure of copolymers were determined based on copolymerization constants [16]. The prepared data are presented in Table 1.

The constant values of relative activity (Table 1) evidence about large reactivity of PCPC in comparison with MMA, which has been connected with influence of substituent of cyclopropane ring on electron density of double bond of vinyl group [17]. The ester group is included in general system of conjugation, causing redistribution of electron density both in monomer and in radical center forming from it. As a result the energy necessary for occurrence of the transition state is decreased leading to the increase of reactivity of monomer.

The calculated parameters value $\mathrm{Q}_{1}$ and $\mathrm{e}_{1}$ at copolymerization with MMA indicate to the increased conjugation in the monomer (PCPC) connected with influence of substituent - cinnamate fragment, stipulating high reactivity of monomer and more low reactivity of radicals. In calculation of factor $e_{1}$ it was chosen a negative sign, based on that the electron density of double bond of the vinyl group PCPC should be less than that of MMA, since an influence of substituents at PCPC leads to a redistribution of the electron cloud density at double bond of the vinyl group changing a radical polarity. A larger reactivity of $\mathrm{PCPC}$ in comparison with MMA confirmed by copolymerization constants $\left(\mathrm{r}_{1}=1.05\right.$, $r_{2}=0.4$, respectively) has been connected with this. On the basis of the calculated copolymerization constants the data of microstructure of copolymers have been prepared (Table 1). A length of the blocks $\mathrm{L}_{\mathrm{M}_{1}}$ is increased with increase of PCPC fraction in the composition of copolymer. It is seen from Table that $\mathrm{R}$ and $\mathrm{L}_{\mathrm{M}_{1}}$ are maximal (58 and 38; 2.05 and 4.15 units, respectively) at ratio of the initial monomers 50:50, 75:25. Hence it follows that by selection of the determined compositions of monomer mixtures one can realize a directed formation of microstructure of the copolymers which is one of the most perspective ways of modification of their properties.

An availability of reactive grouping of various chemical nature in links of macromolecule of the synthesized copolymer causes an interest to the investigation of photochemical structuring of this copolymer, i.e. cross-linking under action of UV-irradiation.

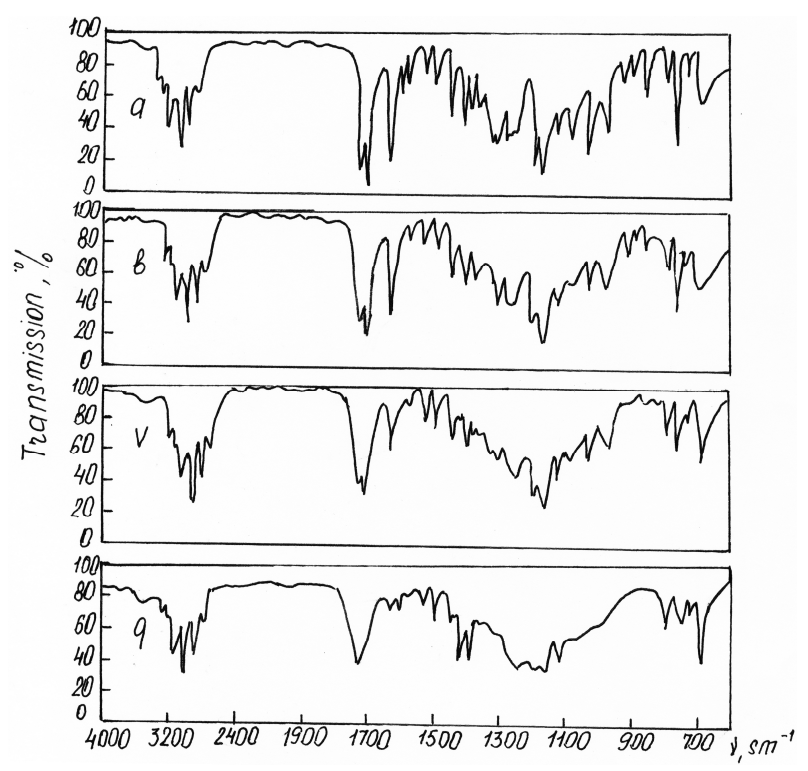

Fig. 1. IR-spectra of film of copolymer of PCPC with MMA : non-irradiated (a) and irradiated for 1 (b), 3 (c) and 4 (d) minutes. $m_{1}: m_{2}=78.54: 21.46$ mol\%.

The photochemical investigations of the synthesized copolymers were carried out according to the methodology [10]. Owing to availability of groups strongly-absorbing light energy (cyclopropane, double bonds, carbonyl) the synthesized copolymers are photosensitive and under action by UV-irradiation are subjected to the photochemical conversions leading to the formation of net structures. By the IR-spectroscopic investigations the process of structuring of the prepared cyclopropane-containing copolymers has been studied. Depending on irradiation duration (20-25 min.) an 
intensity of maximums of the absorption bands characteristic for cyclopropane ring $\left(1030-1035 \mathrm{~cm}^{-1}\right)$, double bond $(1635$ $\mathrm{cm}^{-1}$ ) and carbonyl group (1720-1725 $\left.\mathrm{cm}^{-1}\right)$, considerably falls or completely disappears (see fig. 1).

A structuring proceeds due to opening of double bond and cyclopropane ring, carbonyl group in cinnamate fragments.

The absorption band at $1720 \mathrm{~cm}^{-1}$, characteristic for carbonyl group in ester fragment and being in conjugation with double bond, after irradiation is displaced to $1740 \mathrm{~cm}^{-1}$, which has been probably connected with reaction of opening of double bond and, as a consequence, with disturbance of conjugation.

In the irradiation process on change of bromine number the content of double bonds in cinnamate fragment was controlled [15].

It has been established that a photosensitivity of copolymer is in dependence on content of PCPC in the copolymer, i.e. with increasing of PCPC fraction in macromolecule a total photosensitivity is increased. Evidence of this fact is presented in Fig. 2.

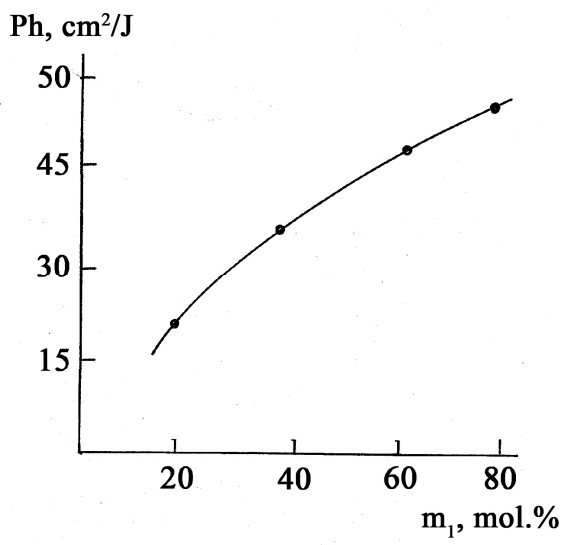

Fig. 2. Dependence of photosensitivity $(P)$ of copolymer of PCPC with MMA on composition.

Thus, an availability of the prepared copolymer of cyclopropane ring, cinnamate and carbonyl groups in macromolecules allowed to create a material with high photosensitivity on the base of copolymer.

As a result of the carried out work the new copolymer has been synthesized and its composition, structure and properties has been established. On the basis of the carried out investigations it can be concluded that the availability of new synthesized copolymer of cyclopropane ring and cinnamate fragment in the structure provides high photosensitivity, creation of solid elastic layer with good adhesion to the substrates and low microdefectness of the polymer films for these copolymers.

\section{References}

[1] Bokov Yu.S. "Photo-, electron- and X-ray resist". Radio and bonds, p.136, 1982.

[2] Filichkina V.N. Khim.promyshlennost za rubezhom (Chem.industry abroad), № 11. pp. 11-27, 1985.

[3] Rupyshev V.G., Ivanko M.P., Kozlova G.I. et al. Plast.massy., № 3. pp. 58-59, 1983.

[4] Tsarev P.K., Baranov V.G., Lipatov Yu.S. Vysokomolek.soyed. (High-molecular compounds), 12Б. pp. 115-117 (1970).

[5] Galaktionov D. Plastics. № 7-8, p.53, 2007.

[6] Vayner A.Ya., Dyumaev K.M. Khim.promyshlennost., № 7. p.3, 1989.

[7] Vayner A.Ya., Dyumaev K.M., Likhachev I.A.et al. Dok1. Akademii Nauk (DAN), 396. № 3. pp. 362-365, 2004.

[8] Hou H., Jiang J., Ding M. Eur. Polym. J, 35. N 11. p. 1993, 1999.

[9] Guliyev K.G., Ponomaryeva G.Z., Guliyev A.M. ZhPKh, 79. № 3. pp. 497-500, 2006.

[10] Guliyev K.G., Ponomaryeva G.Z., Guliyev A.M. Vysokomolek.soyed., 49Б, № 8, pp. 1577-1581, 2007.

[11] Guliyev K.G., Ponomaryeva G.Z., Mamedli S.B. Processes of petrochemistry and oil-refining, 10, № 2. pp. 183-186, 2009.

[12] Guliyev K.G., Ponomaryeva G.Z., Alieva A.M/. Guliyev A.M. ZhPKh, 86, N 1, pp. 101-104, 2013.

[13] Guliyev K.G. ZhPKh, 84, N 12, pp. 2030-2033, 2011.

[14] Guliyev K.G., Ponomaryeva G.Z., Guliyev A.M. Processes of petrochemistry and oil-refining, № 1, pp. 40-43, 2003.

[15] Kuznetsov E.V., Divgun S.M. et al. Practical work on chemistry and physics of polymers. M.: Khimiya, p. 256, 1977.

[16] Zilberman E.N. Vysokomolek.soyed., 215, № 1, pp. 33-36, 1979.

[17] Guliyev K.G., Ponomaryeva G.Z., Mamedli S.B., Guliyev A.M. Journal of Structural Chemistry, 50, № 4, pp. 720-722, 2009. 